\title{
Editorial introduction: Socializing the extended mind
}

Research at the intersection of philosophy and cognitive science has been a dynamically evolving and rapidly growing field that has witnessed increased attention over the last decade. A particular debate that has contributed to this growth concerns the role of non-neural, "external" structures in cognition and the idea that the neural, or even organismic, boundary is a merely arbitrary stopping point for the systematic investigation of cognition. This debate is particularly stimulating for researchers across the traditional disciplinary boundaries as it indicates a gradual change in opinion about how the cognitive apparatus is to be studied. Supporters of this approach have, in various ways, opposed the view that cognition is constituted only by computational, representation-manipulating activity that finds place in the head and have argued instead that the study of cognition must acknowledge the non-trivial role of the body and external structures in cognitive processes.

This special issue expands upon the aforementioned discussion by addressing what the editors and contributors see as lacuna among the extended mind debates. The focus in this issue is on the extent to which "extended" frameworks are useful for understanding the role of social structures in cognition. This is an area of research that has until recently received little attention in the philosophy of cognitive science. The title of this special issue, and of the target paper, refers back to one of the most heavily discussed articles in this field, The Extended Mind (Clark \& Chalmers, 1998) in which the authors introduce the 'Hypothesis of Extended Cognition'. In this paper Clark and Chalmers argue that cognitive activity not only often involves the exploitation of the surrounding environment but that sometimes extra-cranial items actually figure as constituents of cognitive processes. That is to say, proper parts of cognition sometimes extend into the environment. Although this hypothesis has provoked a deluge of opponents and defenders, the debate has tended to focus on the two particular kinds of external items which Clark emphasizes in his works: tools in the environment, and actions of the morphological body. Where social cognition has been considered in the debate, it has typically been in terms of the possibility of using another person as some sort of external memory resource, perhaps betraying the prominent influence of the disciplines of artificial intelligence, computer science and robotics upon the philosophy of cognitive science. In the target paper of this volume Shaun Gallagher takes a quite different tack on this theme of cognitive extension and social cognition by arguing that certain social practices, which he calls "mental institutions", are usefully understood as extending our mind. This proposal could have radical implications for the flow of research between cognitive systems research and the discipline of sociology and pave the way to firmly establishing sociology as one of the cognitive sciences. ${ }^{1}$

The hypothesis of extended cognition, although popular with many, has been far from uncontroversial - even when restricted to the non-social sphere. Since the publication of The Extended Mind, a significant amount of effort has gone into attacking the central thesis that cognitive processes reach beyond the confines of skin and skull and are distributed amongst biological bodies and non-biological tools. Adams and Aizawa $(2010,2005)$ have argued, for example, for the common-sense view that tools such as cellphones, GPS, and notebooks are better understood as having a causal influence upon thoughts, rather than as proper constituents of cognition. Their principal reason for considering these tools as merely instrumental rather than constitutive is that they do not bear the same 'mark' of cognition that brain-endowed cognitions do - whether this 'mark' is conceived to be non-derived content, the ability to informationally update on the spot, or some other stipulated litmus test for genuine thought.

On the other hand, the Hypothesis of Extended Cognition (henceforth 'HEC' in this edition) has garnered support from pro-externalists in the last decade. And Andy Clark himself has continued to press onward in favor of the HEC, though he has modified his original position slightly over the years in response to the attacks (cf. 2008, 2012). Clark's strategy of modifying the position, or better bolstering its central premises, has resulted in an engaging debate, however the volleying back and forth has also begun to seem, at times, a little intractable and unending. No "cure" for the cognitive hiccups, as of yet,

\footnotetext{
${ }^{1}$ Traditionally the disciplines that comprise cognitive science have been considered to be those which make up the "cognitive hexagon" (See H.Gardner 1985 The Mind's New Science). That is, philosophy, psychology, linguistics, artificial intelligence, neuroscience, and anthropology.
} 
has settled the dispute once and for all. To a large extent, this ongoing dispute rests on a particular reading of what has come to be known as the "Parity Principle", and it is worth briefly rehearsing this principle in order to provide context for the upcoming articles. The parity principle was originally stated as follows:

If, as we confront some task, a part of the world functions as a process which, were it done in the head, we would have no hesitation in recognizing as part of the cognitive process, then that part of the world is part of the cognitive process $[1998 ; 11]$

On a strict reading (i.e. taking it as a requirement rather than a guide for our intuitions) the parity principle is interpreted as stating that in order to determine whether a process ought to be considered cognitive we need to assess its functional similarity to processes already clearly known to be cognitive; that is to say "brain-bound" mental processes. So, a corollary question emerges, when considering how to mark cognition: just how broad a conception of functional similarity is acceptable? If, on the other hand, the requirement of maintaining parity between internal brain processes and external world processes is abandoned and we use the principle instead as an intuition pump, the landscape of the debate changes dramatically (see for example Menary, 2010b; Rowlands, 2010; Sutton, 2010). As the editors of this special edition see it, this is an attractive view that could help secure progress. It is partly due to our conviction that the issue of parity has been overplayed that we have compiled a group of papers that, rather than seeking to rehearse, defend, and attack issues pertaining to parity, sidestep that question altogether. Instead of focusing on those sorts of processes traditionally thought to be subtended by brain processes and then examining external processes to determine to what degree parity between the two types is maintained, the authors herein are concerned with the role that social institutions play in shaping - and constituting - certain forms of cognition. The issue of parity, therefore, could turn out to be moot when considering the way the "social mind" works; it might not make sense to search for functional similarity between brain processes and those sorts of socially cognitive processes that are, by their very nature, distributed among various external features in the environment.

The target article in this special issue, "The Socially Extended Mind" argues precisely this point. Gallagher's main thesis is that "[t]he use of various institutional procedures and social practices may offer structures that support and extend our cognitive abilities" (Gallagher, 2013) and he argues that a lack of parity between these socially instituted cognitive processes and brain processes ought not disqualify a system from proper consideration in cognitive science. On the contrary, he claims, by insisting on the criterion of parity, we are most likely overlooking important features of cognition - in this case, its social features - that are inherently dissimilar to any brain processes but nevertheless ought to be considered partially constitutive of cognition. By taking social institutions as genuine constituents of thinking, Gallagher argues, cognitive science will be more inclusive of all of cognition, including social cognition. Furthermore, the view that the mind is socially extended will allow for insight into critical theory and its connections to cognition, a promising interdisciplinary investigation for which an otherwise brain-bound cognitive science would have little to offer.

The collection of papers assembled in this special issue responds to and develops Gallagher's target paper on the Socially Extended Mind. The contributions represent a broad range of thinking about the boundaries and the nature of cognition, but they can roughly be grouped into three categories. Some of the authors are critical of the whole approach, some are more sympathetic to this particular idea of social externalism, but disagree with parts of Gallagher's argument, and others focus on developing further the idea of social institutions. Huebner's contribution belongs to the first category, and his argument is perhaps the most critical of our collection, as he sets out to maintain an internalist and representationalist account of cognition. He thereby not only rejects Gallagher's suggestion that mental institutions carry cognitive processes, but also that any external system or tool could do so. While Huebner maintains that our cognitive processes are interestingly embedded in social practices, he argues that these are not genuine constituents of cognition, but rather, are akin to how Adams and Aizawa think we ought to consider Otto's notebook; social institutions aide in the cognitive process, but are not a part of the process itself.

Although they come from very different vantage points, both De Jaegher's and Menary's contributions agree with Gallagher's critique of the representationalist framework, but are skeptical with regards to socially extended cognition. De Jaegher's critique begins by agreeing with Gallagher's claim that we need not maintain a functionalist framework when describing cognition. She disagrees with him, however, when it comes to equating the enactivist view of cognition with a socially extended view. Specifically, she argues that the extended view will fall short of capturing the ways in which certain shared meaning-making activities occur, are maintained and are transformed by social institutions. Similarly, Menary also thinks that extended or distributed cognition is not the best route to take in this regard. Rather, he favors an account of "enculturated cognition" that takes seriously not only the embodied and embedded nature of cognition, but also its connection to culture, society, and technology. He argues that this account allows for exploring the role of the environment, sociologically and biologically, without being faced with the challenges that Gallagher's view must confront.

Contrary to the previous contributions, Tollefsen, Dale \& Olsen are sympathetic to the idea of externalism in general, but critical of an ambiguity the they find in Gallagher's argument. More specifically, they argue that the term 'system' itself is unclear, which thereby renders 
it difficult to properly characterize agent-system interaction, a necessary component of Gallagher's argument that cognition and social institutions are coextensive. Drawing on systems theory, the authors suggest ways to clarify levels of description, system-agent engagement, and systems analysis, so as to strengthen the case for a socially extended mind.

The last group of contributions may be characterized by their focus on further developing the idea of social institutions. Krueger explores the link to developmental psychology, and argues that the mere presence of others, especially in early infancy, serves as a kind of social institution that scaffolds cognition. By taking a Vygotskyan approach, Krueger maintains that the actual physical interventions of caregivers "constrain, transform, and ultimately extend the infants' rudimentary social-cognitive capacities" (2013). While Krueger connects the socially extended mind to psychology, Merritt, Varga and Cash develop the idea in ways that could become useful for (critical) social analysis. They all expound on the latter part of Gallagher's paper, in which he claims that we should adapt the socially extended view of cognition to certain modes of critical inquiry. Merritt analyses how social institutions might subtend cognition, and argues that gender ought to be considered an institution in its own right. The social practices, roles, contexts, and performative dynamics that serve to maintain the institution of gender, she claims, are pervasive enough to warrant thinking of gender as a nearly ubiquitous institution through which our cognition is constituted. Specifically, she examines the way sexual pleasure is gendered and pathologized by media and medical science, which thereby inhibits or facilitates, but most certainly constitutes, a particular kind of sexual-cognitive experience.

Varga begins by clarifying some ambiguities he finds in Gallagher's paper, in particular, two uses of the term 'constitution,' and he shows why, on a particular interpretation, Gallagher's notion of constitution is not susceptible to many of the traditional worries surrounding externalist arguments. He then distinguishes between local and global frames of cognition in order to make a case that when thinking about war, for example, within a 'local frame,' our cognitive processes related to legality, justice, peace and so forth, are extended, and sometimes distorted by that specific social framework. All of this, he argues, sheds light on the interplay between cognitive science and critical theory.

In the final contribution to this special issue, Cash criticizes Gallagher's socially extended mind hypothesis for retaining the individual-centeredness common to both internalists and the externalists of the first- and secondwaves (pro-extended mind and pro-integrationist respectively). Cash argues that third-wave accounts of cognition, which see cognition as fundamentally distributed across social and cultural practices, are not individual-centered and it therefore makes no sense to talk of cognition as "extending" from that individual, nor indeed of boundaries of cognition at all. Drawing on feminist relational theory, he argues that this is consistent with understanding ourselves as intelligent agents. Instead of asking questions about whether cognition extends, we should ask questions about responsibility: is the individual responsible for the ideas and actions which come about as a result of their engagement with the social and cultural practices in which they are deeply embedded? In answering such questions we come to an agreement about what ought to be considered 'mine', a notion that differs importantly from Gallagher's appeal to ownership, and which, Cash argues, encompasses all that was key to the original question of where the borders of cognition lie.

Overall, this special edition on Socially Extended Cognition aims to critically engage with the debate concerning where the mind ends and the rest of the world begins. What makes this collection of papers unique is that rather than run through the same tried and tested arguments for and against the original HEC, we have launched an entirely novel way of conceiving what cognitive extension might look like, namely, through the social world and its institutions. Gallagher's account supposedly sidesteps all the typical concerns that pertain to the HEC. What we have tried to do in compiling the papers that follow Gallagher's is provide an assessment of just how successful his argument is in accounting for social-cognitive processes that reach beyond the confines of our biological brains.

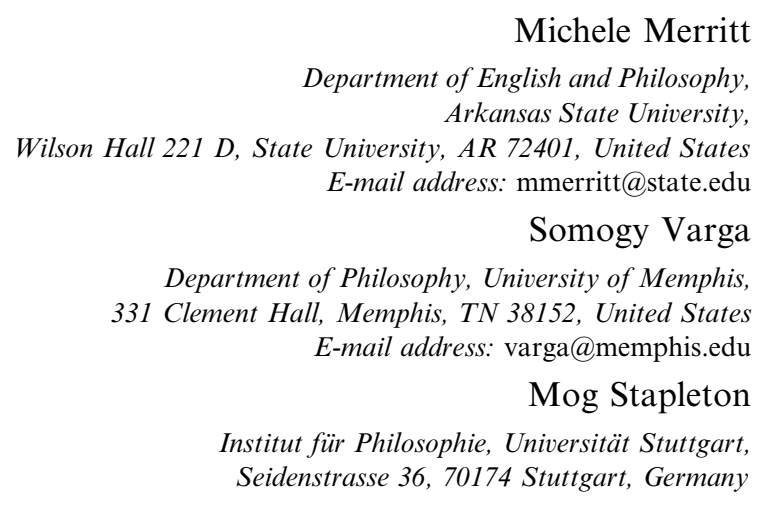

Available online 15 March 2013 